\title{
HY5: A Pivotal Regulator of Light-Dependent Development in Higher Plants
}

\author{
Yuntao Xiao, Li Chu, Yumeng Zhang, Yeting Bian, Jiahui Xiao and Dongqing Xu*
}

State Key Laboratory of Crop Genetics and Germplasm Enhancement, National Center for Soybean Improvement, College of Agriculture, Nanjing Agricultural University, Nanjing, China

ELONGATED HYPOCOTYL5 (HY5), a bZIP-type transcription factor, acts as a master regulator that regulates various physiological and biological processes in plants such as photomorphogenesis, root growth, flavonoid biosynthesis and accumulation, nutrient acquisition, and response to abiotic stresses. HY5 is evolutionally conserved in function among various plant species. HY5 acts as a master regulator of light-mediated transcriptional regulatory hub that directly or indirectly controls the transcription of approximately one-third of genes at the whole genome level. The transcription, protein abundance, and activity of HY5 are tightly modulated by a variety of factors through distinct regulatory mechanisms. This review primarily summarizes recent advances on HY5-mediated molecular and physiological processes and regulatory mechanisms on HY5 in the model plant Arabidopsis as well as in crops.

\section{OPEN ACCESS}

Edited by:

Zhaojun Ding,

Shandong University, China

Reviewed by:

Xiaodong $\mathrm{Xu}$,

Henan University, China Hirokazu Tanaka,

Meiji University, Japan

*Correspondence:

Dongqing Xu

dongqingxu@njau.edu.cn

Specialty section:

This article was submitted to

Plant Cell Biology,

a section of the journal

Frontiers in Plant Science

Received: 24 October 2021 Accepted: 17 December 2021 Published: 17 January 2022

Citation:

Xiao Y, Chu L, Zhang Y, Bian Y, Xiao J and Xu D (2022) HY5: A Pivotal Regulator of Light-Dependent Development in Higher Plants.

Front. Plant Sci. 12:800989. doi: 10.3389/fp/s.2021.800989
Keywords: HY5, photomorphogenesis, root growth, nutrient utilization, pigment accumulation

\section{INTRODUCTION}

Plants utilize light as the predominant energy source for photosynthesis. Besides, light signal acts as an essential external factor that mediates a variety of physiological and developmental processes in plants (Paik and Huq, 2019; Song et al., 2020a; Xu, 2020). Plants are continuously exposed to dynamically changing light signals due to the daily and seasonal alternation in natural conditions. The various light signals are perceived by at least five classes of wavelengthspecific photoreceptors including phytochromes (phyA-phyE), cryptochromes (CRY1 and CRY2), phototropins (PHOT1 and PHOT2), F-box containing flavin binding proteins (ZTL, FKF1, and LKP2), and UV-B RESISTANCE LOCUS 8 (UVR8; Paik and Huq, 2019). These photoreceptors are biologically activated by various light signals, subsequently initiating a large scale of transcriptional reprogramming at the whole genome level (Jing and Lin, 2020). Extensive genetic and biochemical studies have established that the ELONGATED HYPOCOTYL5 (HY5), a bZIP-type transcription factor, tightly controls the light-regulated transcriptional alternation. Loss of HY5 function mutant seedlings displays drastically elongated hypocotyls in various light conditions (Oyama et al., 1997), suggesting that HY5 acts downstream of multiple photoreceptors in promoting photomorphogenesis in plants. In addition to inhibiting hypocotyl growth, HY5 regulates other various physiological and developmental processes including root growth, pigment biosynthesis and accumulation, responses to various hormonal signals, and low and high temperatures (Nawkar et al., 2017; van Gelderen et al., 2018; Zhang Y. et al., 2019; Li J. et al., 2020; Marzi et al., 2020; Ortigosa et al., 2020; Yadukrishnan et al., 2020; Bhagat et al., 2021; Wang et al., 2021a,b). This review summarizes the recent advances and progresses on HY5-regulated cellular, physiological, and developmental processes in various plant species. We also highlighted emerging insights regarding the HY5-mediated integration of multiple developmental, external, and internal signaling inputs in the regulation of plant growth. 


\section{HY5 IS EVOLUTIONALLY CONSERVED IN PLANT SPECIES}

HY5 is originally identified as a positive regulator of photomorphogenesis, root gravitropic response, and lateral root development in the model plant Arabidopsis (Oyama et al., 1997). HY5 gene encodes a bZIP-type transcription factor that controls approximately one-third of the expression of genes throughout the whole genome (Lee et al., 2007; Burko et al., 2020b). Extensive studies have revealed that HY5 regulates a variety of developmental processes, responsiveness of various hormonal and environmental signals through divergent but overlapping signaling networks in plants (Gangappa and Botto, 2016; Su et al., 2021). The orthologs of HY5 in distinct plant species are highly conserved in protein structure and function (Figure 1). HY5 from most plant species possess a basic region and a Leucine Zipper Domain responsible for DNA binding and dimerization, respectively, and the others contain an additional RING-finger motif in some plant species such as soybean and pea (Figure 1). These imply HY5 orthologs likely exert common but divergent functions in regulating physiological and developmental processes among various plant species. The HY5 orthologs in various plant species have been shown to mediate multiple light-regulated development and response. The HY5 in Arabidopsis, soybean, pea, apple, moss, tomato, rice, and maize regulate the hypocotyl or stem growth, shade avoidance, and responses to internal signals (e.g., GA and auxin) and external signals (e.g., light, low, and high temperatures) (Oyama et al., 1997; Yamawaki et al., 2011; An et al., 2017b; Burman et al., 2018; Wang et al., 2018; Huai et al., 2020; Lyu et al., 2021). Sweet wormwood, sweet orange, strawberry, pear, peach, tomato, eggplant, and grape HY5 orthologs are involved in the regulation of light-induced flavonoid biosynthesis and accumulation (Loyola et al., 2016; Li J. et al., 2017; Liu et al., 2018; Hao et al., 2019; Huang et al., 2019; Wu et al., 2019; Li Y. et al., 2020; Wang et al., 2020; Zhao et al., 2021; Figure 2). The biochemical functions of HY 5 are conserved in distinct plant species. HY5 acts as a transcription factor that predominantly binds to the $A C G T$ containing cis-element (e.g., G-box and T/G-box) and controls the expression of numerous target genes in response to light signals, which in turn serves to modulate distinct light-regulated physiological and developmental processes in plants.

\section{HY5 IS A CENTRAL REGULATOR OF LIGHT SIGNALING}

Light tightly controls the seedling development including inhibition of hypocotyl growth, promotion of cotyledon expansion, and accumulation of chlorophyll which is totally termed photomorphogenesis. HY5 acts as an essential and indispensable regulator of this developmental process in Arabidopsis. More importantly, its abundance is correlated with seedling photomorphogenesis. A variety of factors converge on HY5, which indirectly or directly controls the expression of over 3,000 genes, to ensure normal seedling photomorphogenic development in response to dynamically changing light signals.
In the darkness, CONSTITUTIVELY
PHOTOMORPHOGENIC 1 (COP1)-SUPPRESSOR OF PHYTOCHROME A-105 (COP1-SPA) E3 ligase complex directly targets HY5 for polyubiquitination and degradation, and thus, the abundance of HY5 remains at an extremely low level (Osterlund et al., 2000; Han et al., 2020). COP1 SUPPRESSOR 1 (CSU1), CSU2, PHYTOCHROME INTERACTING 1 (PIF1), and SIZ1 act as negative regulators of COP1-SPA complex to ultimately maintain HY5 homeostasis in etiolated seedlings (Xu D. et al., 2014; Xu X. et al., 2014; Xu et al., 2015; Lin et al., 2016). On light irradiation, the activity of COP1-SPA is largely suppressed through multiple regulatory mechanisms. The photoreceptors phyA, phyB, CRY1, and CRY2 directly associate with COP1-SPA to disrupt the formation of the COP1-SPA complex (Podolec and Ulm, 2018). Meanwhile, light-activated CRY1, CRY2, and UVR8 compete with HY5 for COP1 binding through conserved Val-Pro (VP) motifs (Lau et al., 2019; Ponnu et al., 2019). As a long strategy, COP1 migrates from the nucleus to the cytoplasm under prolonged light illumination (Han et al., 2020; Ponnu and Hoecker, 2021). Together, all these molecular regulatory mechanisms consequently serve to trigger the appropriate accumulation of HY5 in the light. Accumulated HY5 directly or indirectly regulates the expression of over 3,000 genes, thereby controlling diverse physiological growth and responses to various hormonal and environmental signals (Lee et al., 2007; Burko et al., 2020b).

HY5 together with a group of B-box proteins (BBXs) work in concert to control the expression of numerous downstream target genes as well as multiple molecular and biological events (Figure 3). BBXs function as rate-limiting cofactors in mediating the molecular action of HY5. BBX20, BBX21, BBX22, and BBX23 enhance the transcriptional activation activity of $\mathrm{HY} 5$ by forming heterodimers (Zhang et al., 2017; Bursch et al., 2020), whereas BBX24, BBX25, BBX28, and BBX29 repress HY5 biochemical activity through a similar regulatory mechanism (Gangappa et al., 2013; Lin et al., 2018; Song et al., 2020b). In addition, BBX11 and BBX21 directly bind to the promoter regions of HY5 to activate its transcription, while HY5 positively regulates the expression of $B B X 11, B B X 21$, and itself, thus forming a transcriptional feedback loop in controlling downstream target gene expression (Xu D. et al., 2016, 2018; Zhao et al., 2020; Job and Datta, 2021; Song et al., 2021). These findings suggest that a subgroup of BBXs and HY5 forms a complex transcriptional network that orchestrates the expression of light-responsive genes. Furthermore, HY5 positively controls $B B X 4$ and $B B X 22$, while negatively controls $B B X 30$ and $B B X 31$ at the transcriptional level (Chang et al., 2008; Heng et al., 2019; Yadav et al., 2019; Liu et al., 2021), suggesting that HY5 also modulates the function of some BBXs by regulating their transcript levels in the light. BBX-HY5 regulatory module likely plays a critical role in the regulation of the expression of numerous light-responsive genes, through which HY5 controls diverse light-dependent development in plants.

In addition to BBXs, other components also mediate the light signal transduction by modulating HY5 activity and/or transcription. TEOSINTE 
BRANCHED1/CYCLOIDEA/PROLIFERATING

CELL

FACTOR (TCP2) and SHI-RELATED SEQUENCE 5 (SRS5) positively control the transcription of HY5 to promote photomorphogenesis (He et al., 2016; Yuan et al., 2018). INOSITOL REQUIRING 80 (INO80) affects the chromatin modifications of HY5 and represses its transcription to inhibit photomorphogenic development (Yang C. et al., 2020). COLD REGULATED 27 (COR27) and COR28 directly interact with HY5 to enhance its transcriptional activation activity toward downstream target genes, consequently leading to the promotion of photomorphogenic development (Li X. et al., 2020; Zhu et al., 2020). HY5 associates with HISTONE DEACETYLASE 15 (HDA15) and recruits it to the promoter regions of target genes, thereby decreasing the levels of histone $\mathrm{H} 4$ acetylation in a light-dependent manner and repressing their transcription (Zhao et al., 2019). Similarly, HY5 recruits the SWI-INDEPENDENT3 LIKE (SNL)-HDA19 deacetylase complex to the chromatin regions of $B B X 22$ and itself, which in turn, decreases the accessibility and histone acetylation and suppresses their transcription (Jing et al., 2021). MYC2, MYC3, and MYC5 bind to E-box cis-element present in the HY5 promoter to activate its expression, while HY5 inhibits the expression of MYC2, suggesting that MYCs and HY5 likely form a negative feedback loop in the regulation of seedling development (Chakraborty et al., 2019; Yi et al., 2020). These results indicate that plants acquired a complicated but delicate regulatory mechanism to fine-tune the HY5 transcript level and activity in the control of photomorphogenesis.

HY5 directly binds to the G-box cis-element present in TANDEM ZINC-FINGER/PLUS3 (TZP) promoter to activate its expression in the far-red light. In turn, TZP competes with COP1 for binding of HY5, thus leading to the accumulation of HY5 that promotes phyA signaling (Li C. et al., 2021). Blue light-activated CRY1 competes with AGB1 for binding of HY5, thus leading to the enhanced biochemical activity of HY5 in promoting photomorphogenesis (Lian et al., 2018). Meanwhile, CRY1 interacts with SWC6 and ARP6 and stabilizes HY5 in blue light. Stabilized HY5 recruits SWR1 complex to HY5 target loci to regulate the transcription of its target genes and photomorphogenesis (Mao et al., 2021b). In response to UV-B light signals, WRKY DNABINDING PROTEIN 36 (WRKY36) represses the HY5 at the transcriptional level to inhibit photomorphogenesis (Yang et al., 2018). These results suggest that distinct light signals perceived by different wavelength-specific photoreceptors modulate the seedling growth at least in part through HY5 and HY5mediated signaling.

\section{HY5 CONTROLS LIGHT-MEDIATED ROOT GROWTH}

Although roots grow in the soil, light signaling transduced from shoot to root affects lateral and primary root development in plants (Yang and Liu, 2020). Light triggers the accumulation of HY5 that positively regulates root growth and development under soil-grown conditions (Lee et al., 2016b;
Zhang Y. et al., 2019). HY1 activates the transcription of HY5 in the root cells. Subsequently, HY5 promotes the accumulation of plant phytohormone auxin in the oscillation zone, leading to lateral growth and branching (Duan et al., 2021). Lightinduced HY5 in the root cells activates the transcription of LAZY4 to promote root gravitropism (Yang P. et al., 2020). Far-red, red, and blue light perceived by PHYs and CRYs in the shoot regulate lateral and primary root growth through the HY5 (Lee et al., 2016a; van Gelderen et al., 2018; Gao et al., 2021). HY5 is induced by far-red light in the lateral root primordia in a phytochrome-dependent manner. HY5 decreases the abundance of auxin transporters PIN-FORMED3 and LIKE-AUX1 3 in the plasma membrane to inhibit lateral growth under low red:far-red light conditions (van Gelderen et al., 2018). Red and blue lights stabilize the HY5 dependent on phyB or CRYs in the root, where it activates the miR163 and itself to promote primary root growth (Gao et al., 2021; Li T. et al., 2021). Thus, these results suggest that distinct wavelength-specific photoreceptors transduce the light signals to HY5 in the root cells where it regulates the root growth in the soil.

\section{HY5 REGULATES THE PIGMENT ACCUMULATION}

Anthocyanins are a class of flavonoids that provide protection against biotic and abiotic stresses. HY5 integrates distinct environmental signals such as light, low and high temperatures, salinity, and drought stresses in the control of anthocyanin biosynthesis. HY5 not only directly activates the MYB12 transcription but also directly binds to the promoter regions of multiple anthocyanin biosynthetic genes to activate their transcription (Shin et al., 2007; Bhatia et al., 2021). In addition, HY5 represses the expression of MYB-LIKE 2 (MYBL2), which is a negative regulator of anthocyanin biosynthesis (Nguyen et al., 2015; Wang et al., 2016; Kim et al., 2017). Consequently, these molecular events contribute to anthocyanin biosynthesis and accumulation. In apple, MdHY5 interacts with MdBBX22 to promote the expression of genes involved in anthocyanin biosynthesis (Henry-Kirk et al., 2018; An et al., 2019). Meanwhile, MdHY5 directly activates CONSTANSLIKE 11 (MdCOL11), MdMYBDL1, MdMYB10, and itself but represses MdWRKY41 transcript level, which in turn promote anthocyanin biosynthesis (Bai et al., 2014; An et al., 2017a, 2020; Liu et al., 2019; Mao et al., 2021a). In tomatoes, SlHY5 also controls the expression of anthocyanin biosynthetic genes to promote anthocyanin accumulation (Liu et al., 2018; Qiu et al., 2019). Red pear PyHY5 alone or together with PyBBX18 promotes the expression of PyMYB10 and WD40 PROTEIN GENE (PyWD40), leading to the anthocyanin biosynthesis (Bai et al., 2019; Wang et al., 2020). Moreover, the other HY5 orthologs in multiple plant species such as strawberry, blood orange, grape, peach, and eggplant have also been shown to promote the light-induced anthocyanin biosynthesis and accumulation (Li J. et al., 2017; Huang et al., 2019; Li Y. et al., 2020; Sun et al., 2020; Zhao et al., 2021). 


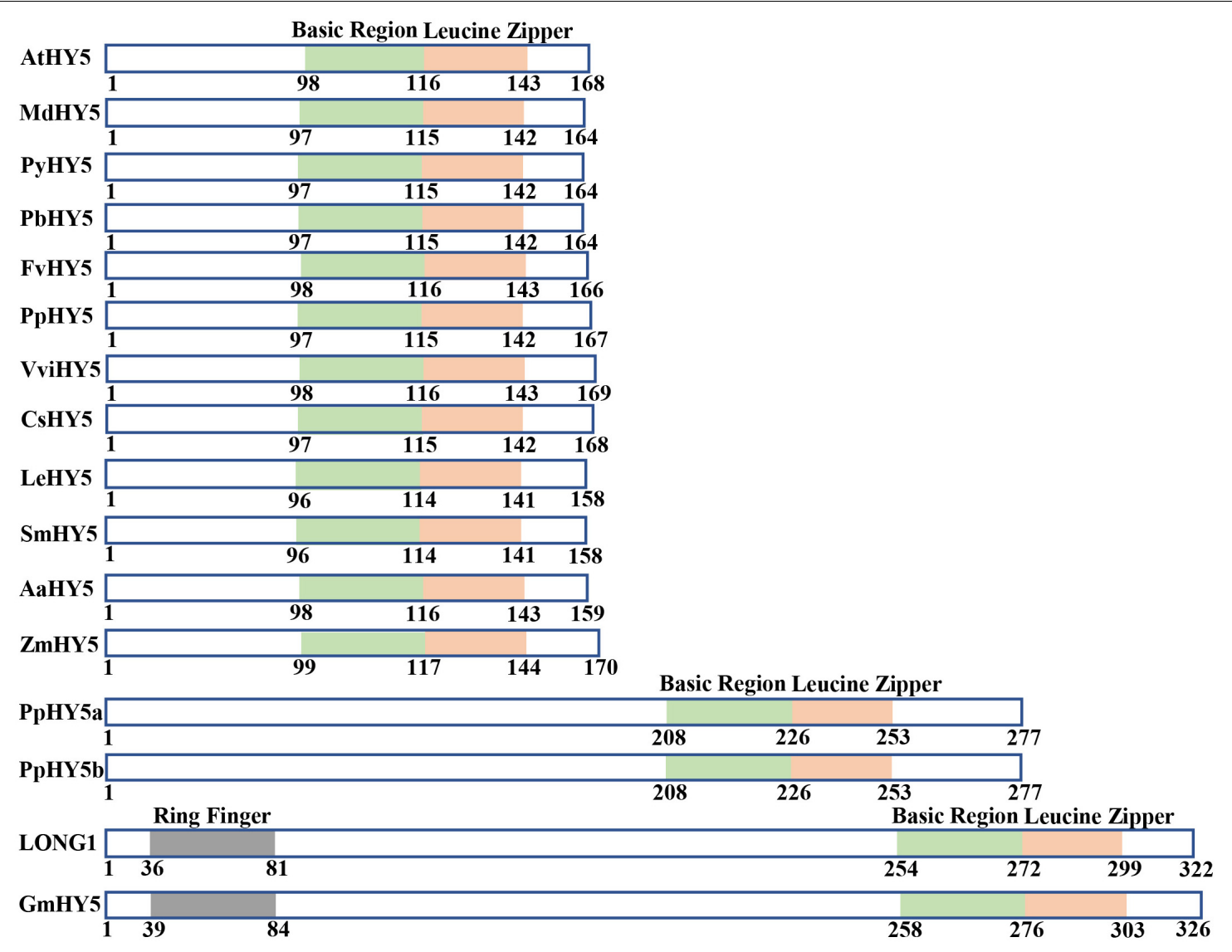

FIGURE 1 | HY5 orthologs are evolutionally conserved in protein structure. Schematic representations of domains present in multiple HY5 orthologs. AtHY5 (Oyama et al., 1997), MdHY5 (An et al., 2017a), PyHY5 (Wang et al., 2020), PbHY5 (Wu et al., 2019), FvHY5 (Li Y. et al., 2020$),$ PpHY5 (Zhao et al., 2017 ), VviHY5 (Loyola et al., 2016), CsHY5 (Huang et al., 2019), LeHY5 (Liu et al., 2004), SmHY5 (Jiang et al., 2016), AaHY5 (Hao et al., 2019$),$ ZmHY5 (Huai et al., 2020$),$ PpHY5a and PpHY5b (Yamawaki et al., 2011), LONG 1 (Weller et al., 2009), and GmHY5 (Song et al., 2008). Numbers indicate the position of amino acid residues.

All these facts suggest that HY5 is an essential regulator of anthocyanin biosynthesis and accumulation, and its function in promoting this physiological process is conserved among diverse plant species.

Besides, light-induced HY5 is also involved in many other secondary metabolite biosynthesis and accumulation in plants. Knock-down LeHY5 transcription leads to reduced carotenoid levels and pale green immature fruits and leaves, indicating that LeHY5 promotes the carotenoid-mediated fruit ripening in tomatoes (Liu et al., 2004). Consistently, LeHY5 regulates the transcription of genes involved in carotenoid, anthocyanin biosynthesis, and ethylene signaling (Wang et al., 2021d). The expression level of PaHY5 in apricot fruit is correlated with the content of carotenoids during the ripening process, indicating that PaHY5 positively controls carotenoid biosynthesis and accumulation in apricot fruit (Zhang L. et al., 2019). In Artemisia annua, AaHY5 activates GLANDULAR TRICHOME-SPECIFIC WRKY1 (AaGSW1) and AaWRKY9 in a light-dependent manner to promote artemisinin biosynthesis (Hao et al., 2019; Fu et al., 2021). HY5 upregulates the expression of TERPENE SYNTHASE 03 (TPS03), a terpene biosynthetic gene, to facilitate the terpenoid biosynthesis in Arabidopsis (Michael et al., 2020). In summary, all these studies suggest that light-controlled HY5 plays a critical role in the regulation of multiple secondary metabolite biosynthesis in different plant species.

\section{HY5 FUNCTIONS IN THE REGULATION OF NUTRIENT UPTAKE AND UTILIZATION}

Nutrient acquisition and utilization is essential and necessary for plant growth and development. HY5 controls the expression of a set of genes involved in nitrogen uptake and transport including NITRATE TRANSPORTER 1.1 (NRT1.1), NITRATE TRANSPORTER 2.1 (NRT2.1), AMMONIUM TRANSPORTER 1, 2 (AMT1;2), NITRATE REDUCTASE 2 (NIA2), and NITRITE REDUCTASE 1 (NIR1; Jonassen et al., 2009; Huang et al., 2015; Chen et al., 2016; Sakuraba and Yanagisawa, 2018). HY5 also activates the expression of two sucrose efflux genes SUCROSE TRANSPORTER 11 (SWEET11) and SUCROSE TRANSPORTER 12 (SWEET12) by directly associating with their promoters (Chen et al., 2016; Sakuraba and Yanagisawa, 2018). It has been shown that HY5 moves from shoot to root, where it promotes root 


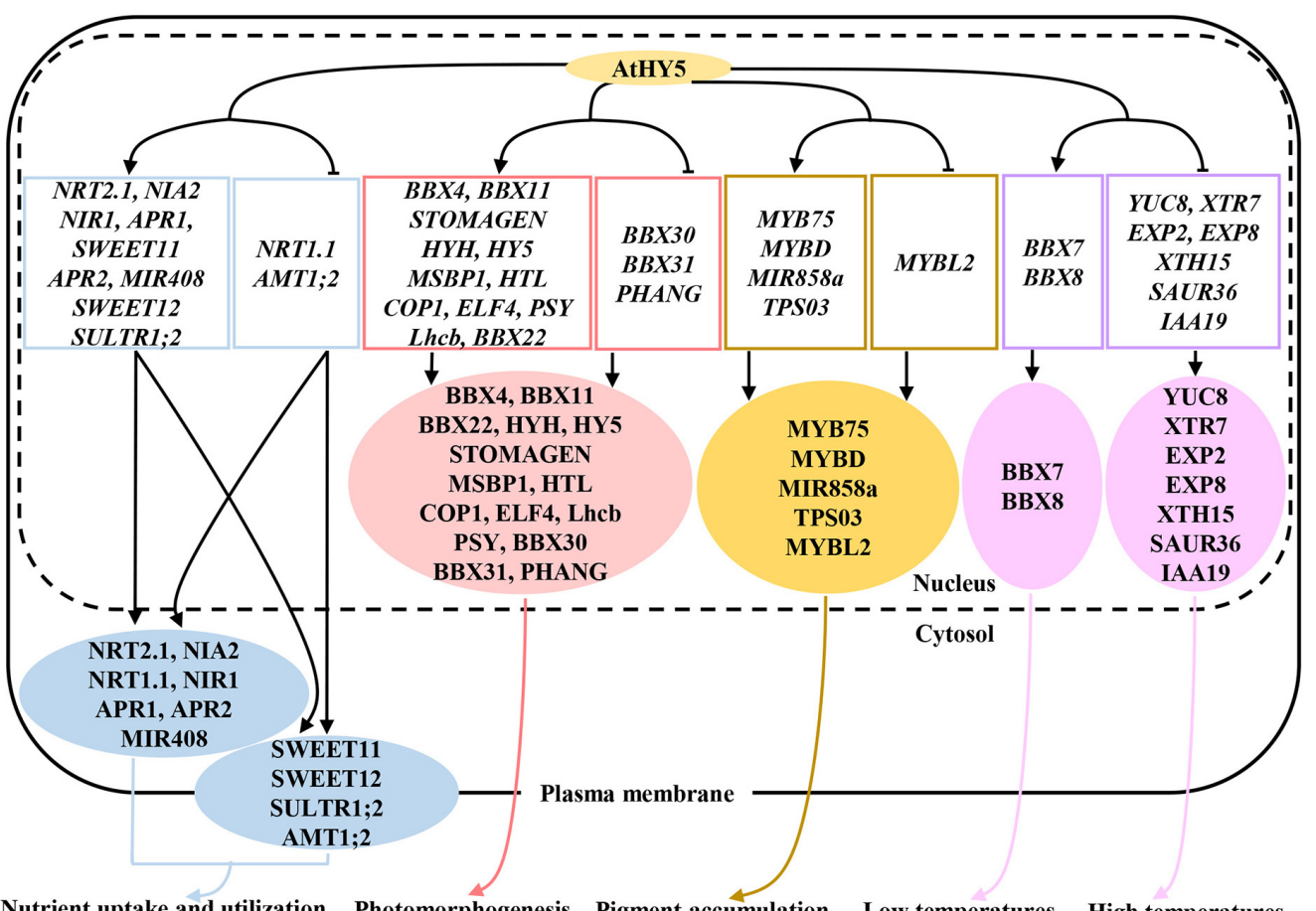

Nutrient uptake and utilization Photomorphogenesis Pigment accumulation Low temperatures High temperatures

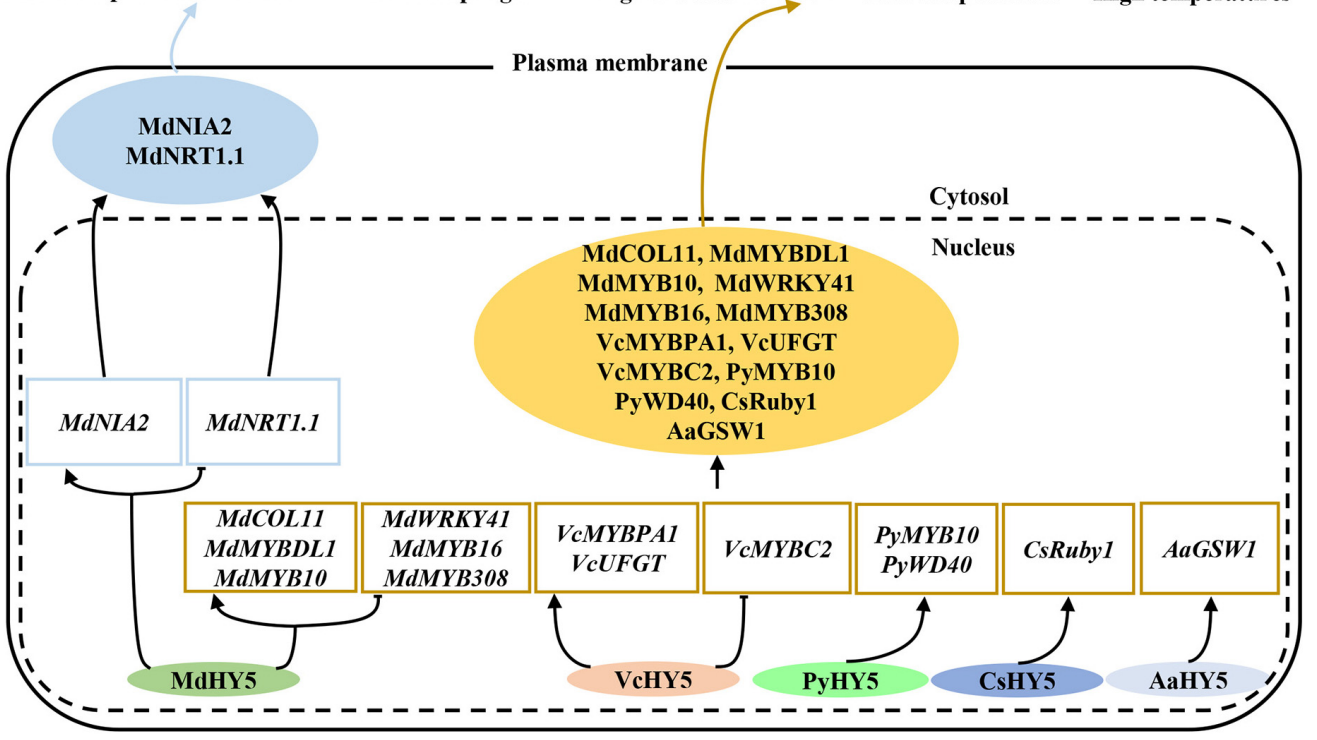

FIGURE 2 | HY5 regulates a variety of physiological and developmental processes in diverse plant species. Various HY5 orthologs are involved in the control of photomorphogenesis, nutrient utilization, pigment accumulation, and low and high temperature signaling by controlling downstream target genes.

growth and nitrate uptake. In the shoot, HY5 facilitates carbon assimilation and translocation, while it activates the nitrate transporter NRT2.1 to enhance nitrate uptake and utilization in the root cells (Chen et al., 2016). A very recent study has shown that HY5 protein mobility is likely not required for shoot-toroot communication. A mobile signal acting downstream of HY5 may function in the shoot-to-root communication (Burko et al., 2020a). Red light activated phyB promotes the accumulation of HY5 both in the shoot and root. A portion of HY5 in the shoot moves to the root, together with root localized HY5, and directly regulates the phosphate starvation-responsive genes to facilitate phosphorus acquisition in Arabidopsis (Sakuraba et al., 2018). SQUAMOSA PROMOTER BINDING PROTEIN-LIKE7 (SPL7) and HY5 act coordinately to regulate the transcription of MIR408 and its target genes, resulting in the alternation of copper allocation to the chloroplast and plastocyanin levels (Zhang et al., 2014).

In apple, MdHY 5 promotes nitrate assimilation by positively regulating the expression of MdNIA2 and MdNRT1.1 (An et al., 2017a). In tomatoes, SIHY5 controls starch degradation and 


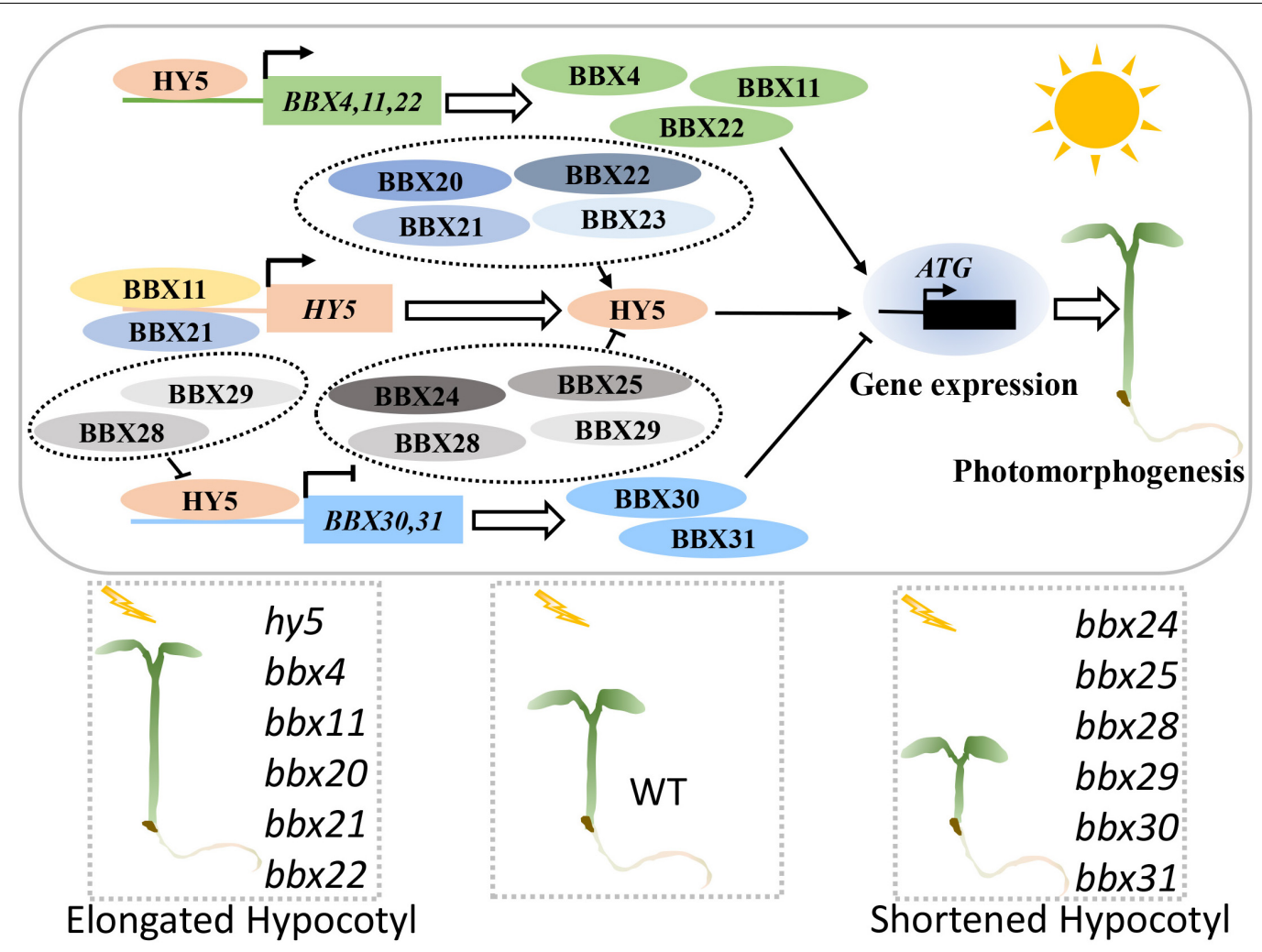

FIGURE 3 | BBX-HY5 regulatory module promotes photomorphogenesis. BBXs and HY5 form a complex transcriptionally regulatory network in promoting photomorphogenesis. HY5 activates the expression of BBX4, BBX11, and BBX22. BBX11 and BBX21 positively control the transcription of $H Y 5$. BBX20, BBX21, BBX22, and BBX23 form heterodimers with HY5 to enhance its activity, whereas BBX24, BBX25, BBX28, and BBX29 interact with HY5 to inhibit its transcriptional activation activity. BBX28 and BBX29 inhibit the HY5 action to upregulate BBX30 and BBX31 at the transcriptional level. Together, these events synergistically serve to control HY5-mediated genes to promote photomorphogenesis. HY5, BBX4, BBX11, BBX20, BBX21, and BBX22 are positive regulators of light signaling, while BBX24, BBX25, BBX28, BBX29, BBX30, and BBX31 negatively regulate photomorphogenesis.

carbon utilization by directly associating with the promoter regions of starch degradation-related genes (e.g., $P W D, B A M 1$, $B A M 3, B A M 8, M E X 1$, and $D P E 1)$ to activate their transcription (Dong et al., 2021). SIphyB promotes light-induced Fe uptake in tomatoes by promoting the accumulation of SIHY5. SIHY5 moves from shoot to root, where it activates the expression of the FER transcription factor, leading to the increase of Fe uptake (Guo et al., 2021). Similarly, red light-activated SIphyB enhances the SIHY5 action in the shoot. Therefore, shoot SIHY5 moves to the root to promote phosphate uptake under phosphate starvation conditions (Ge et al., 2021). Altogether, these results suggest that HY5 is necessary and required for precisely controlling multiple nutrient uptake and utilization in diverse plant species in response to fluctuating light signals (Figure 4).

\section{HY5 MEDIATES THE RESPONSIVENESS OF AMBIENT LOW AND HIGH TEMPERATURES}

As sessile organisms, plants have to cope with the fluctuating temperatures in adapting to daily and seasonal changing cycles. Low temperature is one of the most impactful environmental cues that affect plant growth and development. HY5 controls the expression of approximately $10 \%$ of all cold-induced genes to promote cold acclimation in Arabidopsis. Low temperatures trigger the COP1 translocate from the nucleus to the cytoplasm, thus leading to the inactivation of COP1 and accumulation of HY5 (Catalá et al., 2011). PREFOLDIN 4 (PFD4) accumulates in the nucleus, where it interacts with HY5 to facilitate its polyubiquitination and degradation in a COP1-independent manner in response to low temperatures (Perea-Resa et al., 2017). Low temperatures stabilize phosphorylated blue light photoreceptor CRY2 which competes with COP1 to interact with HY5, thereby allowing the accumulation of HY5 that activates the expression of $B B X 7$ and $B B X 8$. In turn, $B B X 7$ and $B B X 8$ regulate the transcription of a set of cold-responsive genes to promote freezing tolerance in plants (Li Y. et al., 2021). In tomatoes, SIHY5, SIMYB15, and SICBFs work synergistically in response to cold $\left(4^{\circ} \mathrm{C}\right)$. On the one hand, SIHY5 positively regulates SIMYB15 transcription. On the other hand, both SIHY5 and SIMYB15 upregulate the transcript levels of SICBF1, SICBF2, and $S I C B F 3$. Thus, these molecular events increase the cold tolerance in tomatoes (Zhang et al., 2020). MdHY5 and MdMYB108L form a transcriptional feedback loop to promote cold tolerance both dependent and independent on CBF signaling in apple 


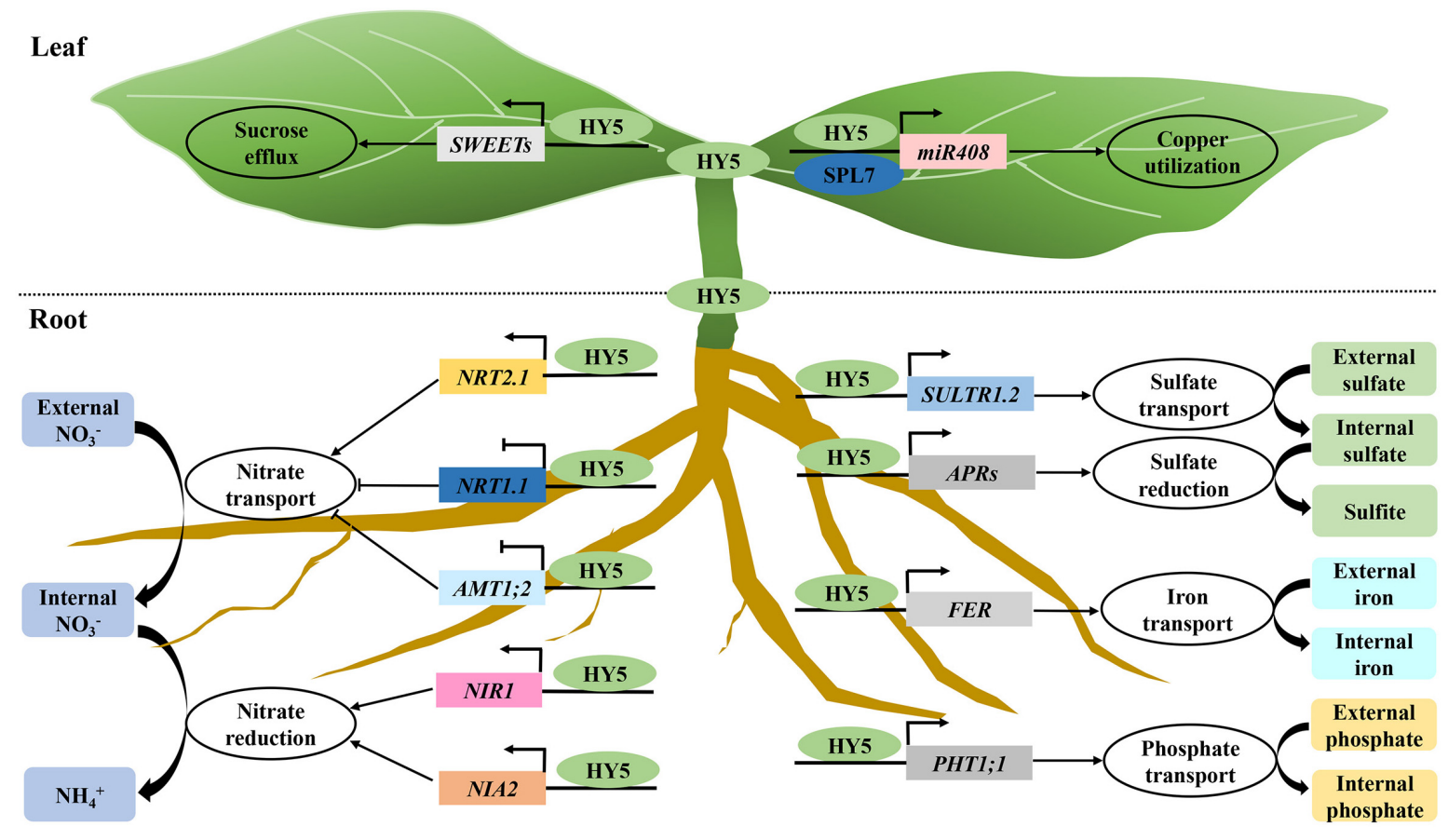

FIGURE 4 | HY5 acts as a central hub of nutrient signaling. Light triggers the accumulation of HY5 both in the shoot and root. HY5 activates the transcription of SWEETS and miR408 to promote sucrose and copper utilization. A portion of shoot localized HY5 proteins move to the root. HY5 proteins in the root regulate the transcription of genes involved in the uptake and/or transport of nitrogen, sulfur, Fe, and phosphate, thereby facilitating multiple nutrient assimilation.

(An et al., 2017b; Wang et al., 2019). Thus, HY5 regulates the cold accumulation both independent of and dependent on CBF signaling in plants.

At elevated high temperatures, HY5 abundance is dramatically reduced due to the inhibition of COP1 activity, resulting in thermomorphogenic development (Kim et al., 2017; Park et al., 2017). In contrast, HY5 competes with PIF4 for repressing PIF4-regulated gene expression and thermomorphogenesis (Gangappa and Kumar, 2017). Shoot and root growth occur simultaneously during early seedling development at high ambient temperatures (Bellstaedt et al., 2019). HY5 is required for controlling root thermomorphogenesis (Gaillochet et al., 2020; Lee et al., 2021). SPA directly phosphorylates HY5 to control its stability, through which HY5 regulates a set of auxin and BR-mediated gene expression in the root cells, consequently promoting root thermomorphogenesis (Lee et al., 2021; Wang et al., 2021c). Therefore, high temperatures tightly control the mode of HY5 action that contributes to both shoot and root thermosensory growth in plants.

\section{CONCLUDING REMARKS AND FUTURE PERSPECTIVES}

Numerous studies have established that HY5 plays pleiotropic roles in regulating various physiological and developmental processes and responses to diverse internal and external signals in plants. A group of components converges on HY5 to modulate its abundance, activity, and transcription in maintaining its appropriate biological action. HY5 acts as a signaling hub that controls the expression of a large number of genes in response to dynamic changing developmental, hormonal, and environmental signals. This mechanistic regulation may ensure the plants adapt to the intracellular and surrounding fluctuating cues throughout their entire life cycles. Increasing studies have shown that HY5 functions are evolutionally conserved among various plant species. The HY5 orthologs in crops control multiple agronomic traits such as stem growth, root growth, nutrient uptake, and fruit ripening. Fulfilling a comprehensive understanding of HY5 functions and signaling will provide novel knowledge and strategies for the improvement of specific agronomic traits in crops. According to current fundamental knowledge on HY5 function and HY5-mediated signaling network, HY5 most likely have positive roles in the control of various physiological and developmental processes. It is therefore loss of HY5 function in different plant species such as Arabidopsis, rice, and soybean, leading to drastically deficient in many facets of development and growth. Increasing HY5 abundance or activity may be a helpful strategy to improve specific agronomic traits in crops. On the one hand, genetic engineering techniques could be applied to generate specific plants expressing appropriately increased HY5 abundance. On the other hand, manipulation of positive or negative regulators of HY5 could be used to enhance the HY5 action. In view of the complexity of HY5 signaling, further studies are required to clarify the detailed HY5 signaling network in diverse plant species. 


\section{AUTHOR CONTRIBUTIONS}

All authors listed have made a substantial, direct, and intellectual contribution to the work, and approved it for publication.

\section{FUNDING}

This study was supported by the Natural Science Foundation of Jiangsu for Distinguished Young Scholars (BK20211525), National Natural Science Foundation of China (31970258),

\section{REFERENCES}

An, J. P., Qu, F. J., Yao, J. F., Wang, X. N., You, C. X., Wang, X. F., et al. (2017a). The bZIP transcription factor MdHY5 regulates anthocyanin accumulation and nitrate assimilation in apple. Hortic. Res. 4:17023.

An, J. P., Yao, J. F., Wang, X. N., You, C. X., Wang, X. F., and Hao, Y. J. (2017b). MdHY5 positively regulates cold tolerance via CBF-dependent and CBF-independent pathways in apple. J. Plant Physiol. 218, 275-281. doi: 10. 1016/j.jplph.2017.09.001

An, J. P., Wang, X. F., Espley, R. V., Lin-Wang, K., Bi, S. Q., You, C. X., et al. (2020). An apple B-Box protein MdBBX37 modulates anthocyanin biosynthesis and hypocotyl elongation synergistically with MdMYBs and MdHY5. Plant Cell Physiol. 61, 130-143. doi: 10.1093/pcp/pcz185

An, J. P., Wang, X. F., Zhang, X. W., Bi, S. Q., You, C. X., and Hao, Y. J. (2019). MdBBX22 regulates UV-B-induced anthocyanin biosynthesis through regulating the function of MdHY5 and is targeted by MdBT2 for $26 \mathrm{~S}$ proteasome-mediated degradation. Plant Biotechnol. J. 17, 2231-2233. doi: 10. $1111 /$ pbi. 13196

Bai, S., Saito, T., Honda, C., Hatsuyama, Y., Ito, A., and Moriguchi, T. (2014). An apple B-box protein, MdCOL11, is involved in UV-B- and temperature-induced anthocyanin biosynthesis. Planta 240, 1051-1062. doi: 10.1007/s00425-0142129-8

Bai, S., Tao, R., Yin, L., Ni, J., Yang, Q., Yan, X., et al. (2019). Two B-box proteins, PpBBX18 and PpBBX21, antagonistically regulate anthocyanin biosynthesis via competitive association with Pyrus pyrifolia ELONGATED HYPOCOTYL 5 in the peel of pear fruit. Plant J. 100, 1208-1223. doi: 10.1111/tpj.14510

Bellstaedt, J., Trenner, J., Lippmann, R., Poeschl, Y., Zhang, X., Friml, J., et al. (2019). A mobile auxin signal connects temperature sensing in cotyledons with growth responses in hypocotyls. Plant Physiol. 180, 757-766. doi: 10.1104/pp. 18.01377

Bhagat, P. K., Verma, D., Sharma, D., and Sinha, A. K. (2021). HY5 and ABI5 transcription factors physically interact to fine tune light and ABA signaling in Arabidopsis. Plant Mol. Biol. 107, 117-127. doi: 10.1007/s11103-021-01187-z

Bhatia, C., Gaddam, S. R., Pandey, A., and Trivedi, P. K. (2021). COP1 mediates light-dependent regulation of flavonol biosynthesis through HY5 in Arabidopsis. Plant Sci. 303:110760. doi: 10.1016/j.plantsci.2020.110760

Burko, Y., Gaillochet, C., Seluzicki, A., Chory, J., and Busch, W. (2020a). Local HY5 activity mediates hypocotyl growth and shoot-to-root communication. Plant Commun. 1:100078. doi: 10.1016/j.xplc.2020.100078

Burko, Y., Seluzicki, A., Zander, M., Pedmale, U. V., Ecker, J. R., and Chory, J. (2020b). Chimeric activators and repressors define HY5 activity and reveal a light-regulated feedback mechanism. Plant Cell 32, 967-983. doi: 10.1105/tpc. 19.00772

Burman, N., Bhatnagar, A., and Khurana, J. P. (2018). OsbZIP48, a HY5 transcription factor ortholog, exerts pleiotropic effects in light-regulated development. Plant Physiol. 176, 1262-1285. doi: 10.1104/pp.17.00478

Bursch, K., Toledo-Ortiz, G., Pireyre, M., Lohr, M., Braatz, C., and Johansson, H. (2020). Identification of BBX proteins as rate-limiting cofactors of HY5. Nat. Plants 6, 921-928. doi: 10.1038/s41477-020-0725-0

Catalá, R., Medina, J., and Salinas, J. (2011). Integration of low temperature and light signaling during cold acclimation response in Arabidopsis. Proc. Natl. Acad. Sci. U.S.A. 108, 16475-16480. doi: 10.1073/pnas.1107161108
Jiangsu "Innovative and Entrepreneurial Talent" Program (to DX), Jiangsu Collaborative Innovation Center for Modern Crop Production (to DX), and Nanjing Agricultural University (startup funding to DX).

\section{ACKNOWLEDGMENTS}

The authors thank all laboratory members for their valuable comments.

Chakraborty, M., Gangappa, S. N., Maurya, J. P., Sethi, V., Srivastava, A. K., Singh, A., et al. (2019). Functional interrelation of MYC2 and HY5 plays an important role in Arabidopsis seedling development. Plant J. 99, 1080-1097. doi: $10.1111 /$ tpj. 14381

Chang, C. S., Li, Y. H., Chen, L. T., Chen, W. C., Hsieh, W. P., Shin, J., et al. (2008). LZF1, a HY5-regulated transcriptional factor, functions in Arabidopsis de-etiolation. Plant J. 54, 205-219. doi: 10.1111/j.1365-313X.2008.03401.x

Chen, X., Yao, Q., Gao, X., Jiang, C., Harberd, N. P., and Fu, X. (2016). Shoot-toroot mobile transcription factor HY5 coordinates plant carbon and nitrogen acquisition. Curr. Biol. 26, 640-646. doi: 10.1016/j.cub.2015.12.066

Dong, H., Hu, C., Liu, C., Wang, J., Zhou, Y., and Yu, J. (2021). ELONGATED HYPOCOTYL 5 mediates blue light-induced starch degradation in tomato. J. Exp. Bot. 72, 2627-2641. doi: 10.1093/jxb/eraa604

Duan, X., Xu, S., Xie, Y., Li, L., Qi, W., Parizot, B., et al. (2021). Periodic root branching is influenced by light through an HY1-HY5-auxin pathway. Curr. Biol. 31, 3834-3847. doi: 10.1016/j.cub.2021.06.055

Fu, X., Peng, B., Hassani, D., Xie, L., Liu, H., Li, Y., et al. (2021). AaWRKY9 contributes to light- and jasmonate-mediated to regulate the biosynthesis of artemisinin in Artemisia annua. New Phytol. 231, 1858-1874. doi: 10.1111/nph. 17453

Gaillochet, C., Burko, Y., Platre, M. P., Zhang, L., Simura, J., Willige, B. C., et al. (2020). HY5 and phytochrome activity modulate shoot-to-root coordination during thermomorphogenesis in Arabidopsis. Development 147:dev192625. doi: 10.1242/dev.192625

Gangappa, S. N., and Botto, J. F. (2016). The multifaceted roles of HY5 in plant growth and development. Mol. Plant 9, 1353-1365. doi: 10.1016/j.molp.2016. 07.002

Gangappa, S. N., Crocco, C. D., Johansson, H., Datta, S., Hettiarachchi, C., Holm, M., et al. (2013). The Arabidopsis B-BOX protein BBX25 interacts with HY5, negatively regulating BBX22 expression to suppress seedling photomorphogenesis. Plant Cell 25, 1243-1257. doi: 10.1105/tpc.113.109751

Gangappa, S. N., and Kumar, S. V. (2017). DET1 and HY5 control PIF4-mediated thermosensory elongation growth through distinct mechanisms. Cell Rep. 18, 344-351. doi: 10.1016/j.celrep.2016.12.046

Gao, Y. Q., Bu, L. H., Han, M. L., Wang, Y. L., and Li, Z. Y. (2021). Longdistance blue light signalling regulates phosphate deficiency-induced primary root growth inhibition. Mol. Plant 14, 1539-1553. doi: 10.1016/j.molp.2021.06. 002

Ge, S., He, L., Jin, L., Xia, X., Li, L., Ahammed, G. J., et al. (2021). Light-dependent activation of HY5 promotes mycorrhizal symbiosis in tomato by systemically regulating strigolactone biosynthesis. New Phytol. [Epub ahead of print]. doi: $10.1111 /$ nph.17883

Guo, Z., Xu, J., Wang, Y., Hu, C., Shi, K., Zhou, J., et al. (2021). The phyBdependent induction of HY5 promotes iron uptake by systemically activating FER expression. EMBO Rep. 22:e51944. doi: 10.15252/embr.202051944

Han, X., Huang, X., and Deng, X. W. (2020). The photomorphogenic central repressor COP1: conservation and functional diversification during evolution. Plant Commun. 1:100044. doi: 10.1016/j.xplc.2020.100044

Hao, X., Zhong, Y., Nï Tzmann, H. W., Fu, X., Yan, T., Shen, Q., et al. (2019). Lightinduced artemisinin biosynthesis is regulated by the bZIP transcription factor AaHY5 in Artemisia annua. Plant Cell Physiol. 60, 1747-1760. doi: 10.1093/pcp/ pcz084 
He, Z., Zhao, X., Kong, F., Zuo, Z., and Liu, X. (2016). TCP2 positively regulates HY5/HYH and photomorphogenesis in Arabidopsis. J. Exp. Bot. 67, 775-785. doi: $10.1093 / j x b / e r v 495$

Heng, Y., Lin, F., Jiang, Y., Ding, M., Yan, T., Lan, H., et al. (2019). B-Box containing proteins BBX30 and BBX31, acting downstream of HY5, negatively regulate photomorphogenesis in Arabidopsis. Plant Physiol. 180, 497-508. doi: 10.1104/pp.18.01244

Henry-Kirk, R. A., Plunkett, B., Hall, M., McGhie, T., Allan, A. C., Wargent, J. J., et al. (2018). Solar UV light regulates flavonoid metabolism in apple (Malus $x$ domestica). Plant Cell Environ. 41, 675-688. doi: 10.1111/pce.13125

Huai, J., Jing, Y., and Lin, R. (2020). Functional analysis of ZmCOP1 and ZmHY5 reveals conserved light signaling mechanism in maize and Arabidopsis. Physiol. Plant 169, 369-379. doi: 10.1111/ppl.13099

Huang, D., Yuan, Y., Tang, Z., Huang, Y., Kang, C., Deng, X., et al. (2019). Retrotransposon promoter of Rubyl controls both light- and cold-induced accumulation of anthocyanins in blood orange. Plant Cell Environ. 42, 30923104. doi: 10.1111/pce.13609

Huang, L., Zhang, H., Zhang, H., Deng, X. W., and Wei, N. (2015). HY5 regulates nitrite reductase 1 (NIR1) and ammonium transporter1;2 (AMT1;2) in Arabidopsis seedlings. Plant Sci. 238, 330-339. doi: 10.1016/j.plantsci.2015. 05.004

Jiang, M., Ren, L., Lian, H., Liu, Y., and Chen, H. (2016). Novel insight into the mechanism underlying light-controlled anthocyanin accumulation in eggplant (Solanum melongena L.). Plant Sci. 249, 46-58. doi: 10.1016/j.plantsci.2016.04. 001

Jing, Y., Guo, Q., and Lin, R. (2021). The SNL-HDA19 histone deacetylase complex antagonizes HY5 activity to repress photomorphogenesis in Arabidopsis. New Phytol. 229, 3221-3236. doi: 10.1111/nph.17114

Jing, Y., and Lin, R. (2020). Transcriptional regulatory network of the light signaling pathways. New Phytol. 227, 683-697. doi: 10.1111/nph.16602

Job, N., and Datta, S. (2021). PIF3/HY5 module regulates BBX11 to suppress protochlorophyllide levels in dark and promote photomorphogenesis in light. New Phytol. 230, 190-204. doi: 10.1111/nph.17149

Jonassen, E. M., Sévin, D. C., and Lillo, C. (2009). The bZIP transcription factors $\mathrm{HY} 5$ and $\mathrm{HYH}$ are positive regulators of the main nitrate reductase gene in Arabidopsis leaves, NIA2, but negative regulators of the nitrate uptake gene NRT1.1. J. Plant Physiol. 166, 2071-2076. doi: 10.1016/j.jplph.2009.05.010

Kim, S., Hwang, G., Lee, S., Zhu, J. Y., Paik, I., Nguyen, T. T., et al. (2017). High ambient temperature represses anthocyanin biosynthesis through degradation of HY5. Front. Plant Sci. 8:1787. doi: 10.3389/fpls.2017.01787

Lau, K., Podolec, R., Chappuis, R., Ulm, R., and Hothorn, M. (2019). Plant photoreceptors and their signaling components compete for COP1 binding via VP peptide motifs. EMBO J. 38:e102140. doi: 10.15252/embj.2019102140

Lee, H. J., Ha, J. H., Kim, S. G., Choi, H. K., Kim, Z. H., Han, Y. J., et al. (2016a). Stem-piped light activates phytochrome B to trigger light responses in Arabidopsis thaliana roots. Sci. Signal. 9:ra106. doi: 10.1126/scisignal.aaf6530

Lee, H. J., Ha, J. H., and Park, C. M. (2016b). Underground roots monitor aboveground environment by sensing stem-piped light. Commun. Integr. Biol. 9:e1261769. doi: 10.1080/19420889.2016.1261769

Lee, J., He, K., Stolc, V., Lee, H., Figueroa, P., Gao, Y., et al. (2007). Analysis of transcription factor HY5 genomic binding sites revealed its hierarchical role in light regulation of development. Plant Cell 19, 731-749. doi: 10.1105/tpc.106. 047688

Lee, S., Wang, W., and Huq, E. (2021). Spatial regulation of thermomorphogenesis by HY5 and PIF4 in Arabidopsis. Nat. Commun. 12:3656. doi: 10.1038/s41467021-24018-7

Li, C., Qi, L., Zhang, S., Dong, X., Jing, Y., Cheng, J., et al. (2021). Mutual upregulation of HY5 and TZP in mediating phytochrome A signaling. Plant Cell [Epub ahead of print]. doi: 10.1093/plcell/koab254

Li, J., Ren, L., Gao, Z., Jiang, M., Liu, Y., Zhou, L., et al. (2017). Combined transcriptomic and proteomic analysis constructs a new model for lightinduced anthocyanin biosynthesis in eggplant (Solanum melongena L.). Plant Cell Environ. 40, 3069-3087. doi: 10.1111/pce.13074

Li, J., Terzaghi, W., Gong, Y., Li, C., Ling, J. J., Fan, Y., et al. (2020). Modulation of BIN2 kinase activity by HY5 controls hypocotyl elongation in the light. Nat. Commun. 11:1592. doi: 10.1038/s41467-020-15394-7

Li, T., Lian, H., Li, H., Xu, Y., and Zhang, H. (2021). HY5 regulates lightresponsive transcription of microRNA163 to promote primary root elongation in Arabidopsis seedlings. J. Integr. Plant Biol. 63, 1437-1450. doi: 10.1111/jipb. 13099

Li, X., Liu, C., Zhao, Z., Ma, D., Zhang, J., Yang, Y., et al. (2020). COR27 and COR28 are novel regulators of the COP1-HY5 regulatory hub and photomorphogenesis in Arabidopsis. Plant Cell 32, 3139-3154. doi: 10.1105/tpc.20.00195

Li, Y., Shi, Y., Li, M., Fu, D., Wu, S., Li, J., et al. (2021). The CRY2-COP1-HY5BBX7/8 module regulates blue light-dependent cold acclimation in Arabidopsis. Plant Cell 33, 3555-3573. doi: 10.1093/plcell/koab215

Li, Y., Xu, P., Chen, G., Wu, J., Liu, Z., and Lian, H. (2020). FvbHLH9 functions as a positive regulator of anthocyanin biosynthesis by forming a HY5-bHLH9 transcription complex in strawberry fruits. Plant Cell Physiol. 61, 826-837. doi: $10.1093 / \mathrm{pcp} / \mathrm{pcaa} 010$

Lian, H., Xu, P., He, S., Wu, J., Pan, J., Wang, W., et al. (2018). Photoexcited CRYPTOCHROME 1 interacts directly with G-Protein $\beta$ Subunit AGB1 to regulate the DNA-binding activity of HY5 and photomorphogenesis in Arabidopsis. Mol. Plant 11, 1248-1263. doi: 10.1016/j.molp.2018.08.004

Lin, F., Jiang, Y., Li, J., Yan, T., Fan, L., Liang, J., et al. (2018). BBOX DOMAIN PROTEIN 28 negatively regulates photomorphogenesis by repressing the activity of transcription factor HY5 and undergoes COP1mediated degradation. Plant Cell 30, 2006-2019. doi: 10.1105/tpc.18.00226

Lin, X. L., Niu, D., Hu, Z. L., Kim, D. H., Jin, Y. H., Cai, B., et al. (2016). An Arabidopsis SUMO E3 ligase, SIZ1, negatively regulates photomorphogenesis by promoting COP1 activity. PLoS Genet. 12:e1006016. doi: 10.1371/journal. pgen.1006016

Liu, B., Long, H., Yan, J., Ye, L., Zhang, Q., Chen, H., et al. (2021). A HY5-COL3COL13 regulatory chain for controlling hypocotyl elongation in Arabidopsis. Plant Cell Environ. 44, 130-142. doi: 10.1111/pce.13899

Liu, C. C., Chi, C., Jin, L. J., Zhu, J., Yu, J. Q., and Zhou, Y. H. (2018). The bZip transcription factor HY5 mediates CRY1a-induced anthocyanin biosynthesis in tomato. Plant Cell Environ. 41, 1762-1775. doi: 10.1111/pce.13171

Liu, W., Wang, Y., Sun, J., Jiang, H., Xu, H., Wang, N., et al. (2019). MdMYBDL1 employed by MdHY5 increases anthocyanin accumulation via repression of MdMYB16/308 in apple. Plant Sci. 283, 32-40. doi: 10.1016/j.plantsci.2019.01. 016

Liu, Y., Roof, S., Ye, Z., Barry, C., van Tuinen, A., Vrebalov, J., et al. (2004). Manipulation of light signal transduction as a means of modifying fruit nutritional quality in tomato. Proc. Natl. Acad. Sci. U.S.A 101, 9897-9902. doi: 10.1073/pnas.0400935101

Loyola, R., Herrera, D., Mas, A., Wong, D. C., Höll, J., Cavallini, E., et al. (2016). The photomorphogenic factors UV-B RECEPTOR 1, ELONGATED HYPOCOTYL 5, and HY5 HOMOLOGUE are part of the UV-B signalling pathway in grapevine and mediate flavonol accumulation in response to the environment. J. Exp. Bot. 67, 5429-5445. doi: 10.1093/jxb/erw307

Lyu, X., Cheng, Q., Qin, C., Li, Y., Xu, X., Ji, R., et al. (2021). GmCRY1s modulate gibberellin metabolism to regulate soybean shade avoidance in response to reduced blue light. Mol. Plant 14, 298-314. doi: 10.1016/j.molp.2020.11.016

Mao, Z., Jiang, H., Wang, S., Wang, Y., Yu, L., Zou, Q., et al. (2021a). The MdHY5MdWRKY41-MdMYB transcription factor cascade regulates the anthocyanin and proanthocyanidin biosynthesis in red-fleshed apple. Plant Sci. 306:110848. doi: 10.1016/j.plantsci.2021.110848

Mao, Z., Wei, X., Li, L., Xu, P., Zhang, J., Wang, W., et al. (2021b). Arabidopsis cryptochrome 1 controls photomorphogenesis through regulation of H2A.Z deposition. Plant Cell 33, 1961-1979. doi: 10.1093/plcell/koab091

Marzi, D., Brunetti, P., Mele, G., Napoli, N., Calò, L., Spaziani, E., et al. (2020). Light controls stamen elongation via cryptochromes, phytochromes and COP1 through HY5 and HYH. Plant J. 103, 379-394. doi: 10.1111/tpj.14736

Michael, R., Ranjan, A., Kumar, R. S., Pathak, P. K., and Trivedi, P. K. (2020). Lightregulated expression of terpene synthase gene, AtTPS03, is controlled by the bZIP transcription factor, HY5, in Arabidopsis thaliana. Biochem. Biophys. Res. Commun. 529, 437-443. doi: 10.1016/j.bbrc.2020.05.222

Nawkar, G. M., Kang, C. H., Maibam, P., Park, J. H., Jung, Y. J., Chae, H. B., et al. (2017). HY5, a positive regulator of light signaling, negatively controls the unfolded protein response in Arabidopsis. Proc. Natl. Acad. Sci. U.S.A. 114, 2084-2089. doi: 10.1073/pnas.1609844114

Nguyen, N. H., Jeong, C. Y., Kang, G. H., Yoo, S. D., Hong, S. W., and Lee, H. (2015). MYBD employed by HY5 increases anthocyanin accumulation via repression of MYBL2 in Arabidopsis. Plant J. 84, 1192-1205. doi: 10.1111/tpj. 13077 
Ortigosa, A., Fonseca, S., Franco-Zorrilla, J. M., Fernández-Calvo, P., Zander, M., Lewsey, M. G., et al. (2020). The JA-pathway MYC transcription factors regulate photomorphogenic responses by targeting HY5 gene expression. Plant J. 102, 138-152. doi: 10.1111/tpj.14618

Osterlund, M. T., Hardtke, C. S., Wei, N., and Deng, X. W. (2000). Targeted destabilization of HY5 during light-regulated development of Arabidopsis. Nature 405, 462-466. doi: 10.1038/35013076

Oyama, T., Shimura, Y., and Okada, K. (1997). The Arabidopsis HY5 gene encodes a bZIP protein that regulates stimulus-induced development of root and hypocotyl. Genes Dev. 11, 2983-2995. doi: 10.1101/gad.11.22.2983

Paik, I., and Huq, E. (2019). Plant photoreceptors: multi-functional sensory proteins and their signaling networks. Semin. Cell Dev. Biol. 92, 114-121. doi: 10.1016/j.semcdb.2019.03.007

Park, Y. J., Lee, H. J., Ha, J. H., Kim, J. Y., and Park, C. M. (2017). COP1 conveys warm temperature information to hypocotyl thermomorphogenesis. New Phytol. 215, 269-280. doi: 10.1111/nph.14581

Perea-Resa, C., Rodríguez-Milla, M. A., Iniesto, E., Rubio, V., and Salinas, J. (2017). Prefoldins negatively regulate cold acclimation in Arabidopsis thaliana by promoting nuclear proteasome-mediated HY5 degradation. Mol. Plant 10, 791-804. doi: 10.1016/j.molp.2017.03.012

Podolec, R., and Ulm, R. (2018). Photoreceptor-mediated regulation of the COP1/SPA E3 ubiquitin ligase. Curr. Opin. Plant Biol. 45, 18-25. doi: 10.1016/ j.pbi.2018.04.018

Ponnu, J., and Hoecker, U. (2021). Illuminating the COP1/SPA ubiquitin ligase: fresh insights into its structure and functions during plant photomorphogenesis. Front. Plant Sci. 12:662793. doi: 10.3389/fpls.2021. 662793

Ponnu, J., Riedel, T., Penner, E., Schrader, A., and Hoecker, U. (2019). Cryptochrome 2 competes with COP1 substrates to repress COP1 ubiquitin ligase activity during Arabidopsis photomorphogenesis. Proc. Natl. Acad. Sci. U.S.A. 116, 27133-27141. doi: 10.1073/pnas.1909181116

Qiu, Z., Wang, H., Li, D., Yu, B., Hui, Q., Yan, S., et al. (2019). Identification of candidate HY5-dependent and -independent regulators of anthocyanin biosynthesis in tomato. Plant Cell Physiol. 60, 643-656. doi: 10.1093/pcp/ pcy236

Sakuraba, Y., Kanno, S., Mabuchi, A., Monda, K., Iba, K., and Yanagisawa, S. (2018). A phytochrome-B-mediated regulatory mechanism of phosphorus acquisition. Nat. Plants 4, 1089-1101. doi: 10.1038/s41477-018-0294-7

Sakuraba, Y., and Yanagisawa, S. (2018). Light signalling-induced regulation of nutrient acquisition and utilisation in plants. Semin. Cell Dev. Biol. 83, 123-132. doi: 10.1016/j.semcdb.2017.12.014

Shin, J., Park, E., and Choi, G. (2007). PIF3 regulates anthocyanin biosynthesis in an HY5-dependent manner with both factors directly binding anthocyanin biosynthetic gene promoters in Arabidopsis. Plant J. 49, 981-994. doi: 10.1111/ j.1365-313X.2006.03021.X

Song, Y. H., Yoo, C. M., Hong, A. P., Kim, S. H., Jeong, H. J., Shin, S. Y., et al. (2008). DNA-Binding study identifies C-Box and hybrid C/G-Box or C/A-Box motifs as high-affinity binding sites for STF1 and LONG HYPOCOTYL5 proteins. Plant Physiol. 146, 1862-1877. doi: 10.1104/pp.107.113217

Song, Z., Bian, Y., Liu, J., Sun, Y., and Xu, D. (2020a). B-box proteins: pivotal players in light-mediated development in plants. J. Integr. Plant Biol. 62, 1293-1309. doi: 10.1111/jipb.12935

Song, Z., Yan, T., Liu, J., Bian, Y., Heng, Y., Lin, F., et al. (2020b). BBX28/BBX29, HY5 and BBX30/31 form a feedback loop to fine-tune photomorphogenic development. Plant J. 104, 377-390. doi: 10.1111/tpj.1 4929

Song, Z., Heng, Y., Bian, Y., Xiao, Y., Liu, J., Zhao, X., et al. (2021). BBX11 promotes red light-mediated photomorphogenic development by modulating phyB-PIF4 signaling. aBIOTECH 2, 117-130.

Su, C., Wang, Y., Yu, Y., He, Y., and Wang, L. (2021). Coordinative regulation of plants growth and development by light and circadian clock. aBIOTECH 2, 176-189.

Sun, L., Li, S., Tang, X., Fan, X., Zhang, Y., Jiang, J., et al. (2020). Transcriptome analysis reveal the putative genes involved in light-induced anthocyanin accumulation in grape 'Red Globe' (V. vinifera L.). Gene 728:144284. doi: 10. 1016/j.gene.2019.144284

van Gelderen, K., Kang, C., Paalman, R., Keuskamp, D., Hayes, S., and Pierik, R. (2018). Far-red light detection in the shoot regulates lateral root development through the HY5 transcription factor. Plant Cell 30, 101-116. doi: 10.1105/tpc. 17.00771

Wang, F., Wu, N., Zhang, L., Ahammed, G. J., Chen, X., Xiang, X., et al. (2018). Light signaling-dependent regulation of photoinhibition and photoprotection in tomato. Plant Physiol. 176, 1311-1326. doi: 10.1104/pp.17.01143

Wang, S., Zhou, Z., Rahiman, R., Lee, G. S. Y., Yeo, Y. K., Yang, X., et al. (2021a). Light regulates stomatal development by modulating paracrine signaling from inner tissues. Nat. Commun. 12:3403. doi: 10.1038/s41467-021-23728-2

Wang, T. J., Huang, S., Zhang, A., Guo, P., Liu, Y., Xu, C., et al. (2021b). JMJ17WRKY40 and HY5-ABI5 modules regulate the expression of ABA-responsive genes in Arabidopsis. New Phytol. 2230, 567-584. doi: 10.1111/nph.17177

Wang, W., Paik, I., Kim, J., Hou, X., Sung, S., and Huq, E. (2021c). Direct phosphorylation of HY5 by SPA kinases to regulate photomorphogenesis in Arabidopsis. New Phytol. 230, 2311-2326. doi: 10.1111/nph.17332

Wang, W., Wang, P., Li, X., Wang, Y., Tian, S., and Qin, G. (2021d). The transcription factor SlHY5 regulates the ripening of tomato fruit at both the transcriptional and translational levels. Hortic. Res. 8:83. doi: 10.1038/s41438021-00523-0

Wang, Y., Mao, Z., Jiang, H., Zhang, Z., and Chen, X. (2019). A feedback loop involving MdMYB108L and MdHY5 controls apple cold tolerance. Biochem. Biophys. Res. Commun. 512, 381-386. doi: 10.1016/j.bbrc.2019.03.101

Wang, Y., Wang, Y., Song, Z., and Zhang, H. (2016). Repression of MYBL2 by both microRNA858a and HY5 leads to the activation of anthocyanin biosynthetic pathway in Arabidopsis. Mol. Plant 9, 1395-1405. doi: 10.1016/j.molp.2016.07. 003

Wang, Y., Zhang, X., Zhao, Y., Yang, J., He, Y., Li, G., et al. (2020). Transcription factor PyHY5 binds to the promoters of PyWD40 and PyMYB10 and regulates its expression in red pear 'Yunhongli No. 1'. Plant Physiol. Biochem. 154, 665-674. doi: 10.1016/j.plaphy.2020.07.008

Weller, J. L., Hecht, V., Vander Schoor, J. K., Davidson, S. E., and Ross, J. J. (2009). Light regulation of gibberellin biosynthesis in pea is mediated through the COP1/HY5 pathway. Plant Cell 21, 800-813. doi: 10.1105/tpc.108.063628

Wu, M., Si, M., Li, X., Song, L., Liu, J., Zhai, R., et al. (2019). PbCOP1.1 contributes to the negative regulation of anthocyanin biosynthesis in pear. Plants 8:39. doi: $10.3390 /$ plants 8020039

$\mathrm{Xu}, \mathrm{D}$. (2020). COP1 and BBXs-HY5-mediated light signal transduction in plants. New Phytol. 228, 1748-1753. doi: 10.1111/nph.16296

Xu, D., Jiang, Y., Li, J., Holm, M., and Deng, X. W. (2018). The B-Box domain protein BBX21 promotes photomorphogenesis. Plant Physiol. 176, 2365-2375. doi: 10.1104/pp.17.01305

Xu, D., Jiang, Y., Li, J., Lin, F., Holm, M., and Deng, X. W. (2016). BBX21, an Arabidopsis B-box protein, directly activates HY5 and is targeted by COP1 for $26 \mathrm{~S}$ proteasome-mediated degradation. Proc. Natl. Acad. Sci. U.S.A 113, 7655-7660. doi: 10.1073/pnas.1607687113

Xu, D., Lin, F., Jiang, Y., Huang, X., Li, J., Ling, J., et al. (2014). The RING-Finger E3 ubiquitin ligase COP1 SUPPRESSOR1 negatively regulates COP1 abundance in maintaining COP1 homeostasis in dark-grown Arabidopsis seedlings. Plant Cell 26, 1981-1991. doi: 10.1105/tpc.114.124024

Xu, D., Lin, F., Jiang, Y., Ling, J., Hettiarachchi, C., Tellgren-Roth, C., et al. (2015). Arabidopsis COP1 SUPPRESSOR 2 represses COP1 E3 ubiquitin ligase activity through their coiled-coil domains association. PLoS Genet. 11:e1005747. doi: 10.1371/journal.pgen.1005747

Xu, X., Paik, I., Zhu, L., Bu, Q., Huang, X., Deng, X. W., et al. (2014). PHYTOCHROME INTERACTING FACTOR1 enhances the E3 ligase activity of CONSTITUTIVE PHOTOMORPHOGENIC1 to synergistically repress photomorphogenesis in Arabidopsis. Plant Cell 26, 1992-2006. doi: 10.1105/tpc. 114.125591

Yadav, A., Bakshi, S., Yadukrishnan, P., Lingwan, M., Dolde, U., Wenkel, S., et al. (2019). The B-Box-containing microProtein miP1a/BBX31 regulates photomorphogenesis and UV-B protection. Plant Physiol. 179, 1876-1892. doi: 10.1104/pp.18.01258

Yadukrishnan, P., Rahul, P. V., and Datta, S. (2020). HY5 suppresses, rather than promotes, Abscisic Acid-mediated Inhibition of postgermination seedling development. Plant Physiol. 184, 574-578. doi: 10.1104/pp.20.00783

Yamawaki, S., Yamashino, T., Nakanishi, H., and Mizuno, T. (2011). Functional characterization of HY5 homolog genes involved in early light-signaling in Physcomitrella patens. Biosci. Biotechnol. Biochem. 75, 1533-1539. doi: 10.1271/ bbb.110219 
Yang, C., Yin, L., Xie, F., Ma, M., Huang, S., Zeng, Y., et al. (2020). AtINO80 represses photomorphogenesis by modulating nucleosome density and H2A.Z incorporation in light-related genes. Proc. Natl. Acad. Sci. U.S.A. 117, 3367933688. doi: 10.1073/pnas.2001976117

Yang, P., Wen, Q., Yu, R., Han, X., Deng, X. W., and Chen, H. (2020). Light modulates the gravitropic responses through organ-specific PIFs and HY5 regulation of LAZY4 expression in Arabidopsis. Proc. Natl. Acad. Sci. U.S.A. 117, 18840-18848. doi: 10.1073/pnas.2005871117

Yang, Y., Liang, T., Zhang, L., Shao, K., Gu, X., Shang, R., et al. (2018). UVR8 interacts with WRKY36 to regulate HY5 transcription and hypocotyl elongation in Arabidopsis. Nat. Plants 4, 98-107. doi: 10.1038/s41477-017-0099-0

Yang, Y., and Liu, H. (2020). Coordinated shoot and root responses to light signaling in Arabidopsis. Plant Commun. 1:100026. doi: 10.1016/j.xplc.2020. 100026

Yi, R., Yan, J., and Xie, D. (2020). Light promotes jasmonate biosynthesis to regulate photomorphogenesis in Arabidopsis. Sci. China Life Sci. 63, 943-952. doi: 10.1007/s11427-019-1584-4

Yuan, T. T., Xu, H. H., Zhang, Q., Zhang, L. Y., and Lu, Y. T. (2018). The COP1 target SHI-RELATED SEQUENCE5 directly activates photomorphogenesispromoting genes. Plant Cell 30, 2368-2382. doi: 10.1105/tpc.18.00455

Zhang, H., Zhao, X., Li, J., Cai, H., Deng, X. W., and Li, L. (2014). MicroRNA408 is critical for the HY5-SPL7 gene network that mediates the coordinated response to light and copper. Plant Cell 26, 4933-4953. doi: 10.1105/tpc.114.127340

Zhang, L., Jiang, X., Liu, Q., Ahammed, G. J., Lin, R., Wang, L., et al. (2020). The HY5 and MYB15 transcription factors positively regulate cold tolerance in tomato via the CBF pathway. Plant Cell Environ. 43, 2712-2726. doi: 10.1111/ pce. 13868

Zhang, L., Zhang, Q., Li, W., Zhang, S., and Xi, W. (2019). Identification of key genes and regulators associated with carotenoid metabolism in apricot (Prunus armeniaca) fruit using weighted gene coexpression network analysis. BMC Genomics 20:876. doi: 10.1186/s12864-019-6261-5

Zhang, X., Huai, J., Shang, F., Xu, G., Tang, W., Jing, Y., et al. (2017). A PIF1/PIF3HY5-BBX23 transcription factor cascade affects photomorphogenesis. Plant Physiol. 174, 2487-2500. doi: 10.1104/pp.17.00418

Zhang, Y., Wang, C., Xu, H., Shi, X., Zhen, W., Hu, Z., et al. (2019). HY5 contributes to light-regulated root system architecture under a root-covered culture system. Front. Plant Sci. 10:1490. doi: 10.3389/fpls.2019.01490
Zhao, L., Peng, T., Chen, C. Y., Ji, R., Gu, D., Li, T., et al. (2019). HY5 interacts with the histone deacetylase HDA15 to repress hypocotyl cell elongation in photomorphogenesis. Plant Physiol. 180, 1450-1466. doi: 10.1104/pp.19.0 0055

Zhao, X., Heng, Y., Wang, X., Deng, X. W., and Xu, D. (2020). A positive feedback lop of BBX11-BBX21-HY5 promotes photomorphogenic development in Arabidopsis. Plant Commun. 1:100045. doi: 10.1016/j.xplc.2020.10 0045

Zhao, Y., Dong, W., Wang, K., Zhang, B., Allan, A. C., Wang, K., et al. (2017). Differential sensitivity of fruit pigmentation to ultraviolet light between two peach cultivars. Front. Plant Sci. 8:1552. doi: 10.3389/fpls.2017.0 1552

Zhao, Y., Min, T., Chen, M., Wang, H., Zhu, C., Jin, R., et al. (2021). The photomorphogenic transcription factor PpHY5 regulates anthocyanin accumulation in response to UVA and UVB irradiation. Front. Plant Sci. 11:603178. doi: 10.3389/fpls.2020.603178

Zhu, W., Zhou, H., Lin, F., Zhao, X., Jiang, Y., Xu, D., et al. (2020). COLDREGULATED GENE27 integrates signals from light and the circadian clock to promote hypocotyl growth in Arabidopsis. Plant Cell 32, 3155-3169. doi: $10.1105 /$ tpc.20.00192

Conflict of Interest: The authors declare that the research was conducted in the absence of any commercial or financial relationships that could be construed as a potential conflict of interest.

Publisher's Note: All claims expressed in this article are solely those of the authors and do not necessarily represent those of their affiliated organizations, or those of the publisher, the editors and the reviewers. Any product that may be evaluated in this article, or claim that may be made by its manufacturer, is not guaranteed or endorsed by the publisher.

Copyright (C) 2022 Xiao, Chu, Zhang, Bian, Xiao and Xu. This is an open-access article distributed under the terms of the Creative Commons Attribution License (CC BY). The use, distribution or reproduction in other forums is permitted, provided the original author(s) and the copyright owner(s) are credited and that the original publication in this journal is cited, in accordance with accepted academic practice. No use, distribution or reproduction is permitted which does not comply with these terms. 